\title{
Niacin Ameliorates Hypercalciuria and Hyperphosphaturia Due to Glucocorticoid Administration in Rats
}

\author{
Tahoora Shomali and Ali Fakhrzad \\ Department of Basic Sciences, Division of Pharmacology and Toxicology, \\ School of Veterinary Medicine, Shiraz University, Shiraz, Iran
}

Received 2012-04-18, Revised 2013-01-20; Accepted 2013-07-13

\begin{abstract}
Hypercalciuria and hyperphosphaturia are present in long term and high dose regimens of glucocorticoid therapy. This study aims to evaluate the effect of niacin at its pharmacological dose on calcium and phosphate disturbances due to methylprednisolone administration in growing rats. Twenty one rats were randomly divided into three equal groups and treated as follows for 4 weeks: 1-Normal saline (Control); 2Methyl Prednisolone (MP) acetate, $3.5 \mathrm{mg} \mathrm{kg}$ five days a week, SC and 3- MP acetate, $3.5 \mathrm{mg} \mathrm{kg}^{-1}$ five days a week, SC + niacin $200 \mathrm{mg} \mathrm{kg}^{-1}$ daily by oral gavages. At the end of the experiment, serum and urinary calcium and phosphate assays were performed and calcium content of forth lumbar vertebrate and tibia-fibula bone was determined by atomic absorption method. No significant difference observed in serum calcium or phosphate levels among different groups $(p>0.05)$, however an obvious hypercalciuria associated with hyperphosphaturia was present in MP group as compared to control $(\mathrm{p}<0.001)$. Niacin significantly decreased urinary calcium $(\mathrm{p}<0.001)$ and phosphate $(\mathrm{p}=0.005)$ concentrations as compared to MP group. Calcium level was still significantly higher than control $(\mathrm{p}<0.001)$, while phosphate decreased even to a lower level than control $(\mathrm{p}=0.005)$. Calcium content of forth lumbar vertebrate or tibia-fibula bone of rats remained statistically the same among different groups $(\mathrm{p}>0.05)$. Niacin at its pharmacological dose can ameliorate hypercalciuria and hyperphosphaturia due to long term and high dose glucocorticoid administration in growing rats without affecting bone calcium content. The possible clinical importance of this effect needs to be clarified in future studies.
\end{abstract}

Keywords: Glucocorticoid Administration, Niacin, Calcium, Phosphate, Growing Rats, Lumbar Vertebrate, Tibia-Fibula Bone

\section{INTRODUCTION}

Glucocorticoids (GCs) are widely prescribed to treat immune and inflammatory conditions of different organs including eye, skin, joints, blood, gastrointestinal and respiratory tracts, in veterinary as well as human patients. However, using systemic GCs especially in long term and high dose regimens may be associated with multiple side effects among them are changes in calcium and phosphate homeostasis and bone metabolism. Hypercalciuria is a known adverse effect of treatment with systemic GCs (Bentur et al., 2003). Duzen et al. (2012) demonstrated that GC treatment induces hypercalciuria just after starting the treatment until the end of it, which promptly improves by the cessation of therapy.

Hypercalciuria is present in $85.7 \%$ of people with Cushing's disease; dogs with Cushing's syndrome are 10 times more likely to have calcium-containing uroliths than control dogs (Faggiano et al., 2003; Hess et al., 1998). Despite hypercalciuria, plasma ionized calcium was normal in people and dogs with hypercortisolism compared with control subjects (Faggiano et al., 1982; Ramsey et al., 2005). On the other hand, hyperphosphaturia is a common observation following GC therapy or in Cushing's disease (Vrtovsnik et al., 1994). Human Cushing's patients have

Corresponding Author: Tahoora Shomali, Department of Basic Sciences, Division of Pharmacology and Toxicology, School of Veterinary Medicine, Shiraz University, Shiraz, P.O. Box: 71345-1731, Iran 
hypophosphatemia whereas canine patients have elevated serum phosphorus (Smets et al., 2010).

Niacin (Nicotinic acid or vitamin B3), which strongly increases HDL cholesterol levels and has a well-documented anti atherosclerotic effect, has attracted new interest. The discovery of the nicotinic acid receptor GPR109A, which has recently been renamed Hydroxy-Carboxylic Acid (HCA) receptor 2 $\left(\mathrm{HCA}_{2}\right)$ (Offermanns et al., 2011) has led to new research activities into the mechanisms through which nicotinic acid exerts its pharmacological effects (Gille et al., 2008; Kamanna et al., 2009a). Recent studies have shown that the nicotinic acid receptor is expressed in various cells including adipocytes, several types of immune cells and keratinocytes. Evidence suggests that nicotinic acid has lipid-independent anti-inflammatory effects (Wu et al., 2010; Lukasova et al., 2011a). Although it has been demonstrated that niacin lowers serum phosphate in dialysis patients (Muller et al., 2007), the effects of this agent on calcium and phosphate imbalance due to $\mathrm{GC}$ administration has not been clarified yet.

The present study aims to evaluate the effect of niacin on calcium and phosphate disturbances in growing rats treated with Methyl Prednisolone (MP).

\section{MATERIALS AND METHODS}

\subsection{Animals and Experimental Design}

Twenty one female Sprague-Dawley rats with about three weeks of age and a mean body weight of $220 \mathrm{~g}$ were purchased from animal house of Shiraz Medical University, Shiraz, Iran. Rats were acclimatized for one week before the beginning of the experiment to the ambient conditions (temperature about $23^{\circ} \mathrm{C}$ and a $12 \mathrm{~h} / 12 \mathrm{~h}$, light/dark cycle). Animals had free access to tap water and standard rat chow diet prepared by Razi Vaccine and Serum Research Institute, Shiraz, Iran. After adaptation, rats were randomly divided into three equal groups $(n=7$ each) and treated as follows for 4 weeks:

- Normal saline (Control)

- MP acetate (Aburaihan pharmaceutical Co., Tehran, Iran), $3.5 \mathrm{mg} \mathrm{kg}^{-1}$ five days a week, $\mathrm{SC}$

- MP acetate, $3.5 \mathrm{mg} \mathrm{kg}^{-1}$ five days a week, $\mathrm{SC}+$ niacin (Novin Kavosh Mamtir Co., Tehran, Iran) $200 \mathrm{mg} \mathrm{kg}^{-1}$ daily by oral gavages

Procedures used in the present study are in accordance with institutional ethical guidelines of School of Veterinary Medicine, Shiraz University, for care and use of laboratory animals in experiments.

\subsection{Determination of Calcium and Phosphate Levels in Serum and Urine}

At the end of the experiment, rats were fasted over night and voiding urine samples collected in the morning and noon. Blood samples were collected under chloroform anesthesia by cardiac puncture. After centrifugation at $2000 \mathrm{rpm}$ for $20 \mathrm{~min}$, harvested sera were stored in $-70^{\circ} \mathrm{C}$ until use. Calcium and phosphate assays in pooled urinary samples (morning and noon for each animal) and sera were performed by commercial colorimetric kits prepared by Ziest Chem ® Diagnostics, Tehran, Iran.

\subsection{Determination of Calcium Content of Forth Lumbar Vertebrate and Tibia-Fibula Bone}

After blood collection, animals were euthanized by deepening anesthesia. Forth lumbar vertebrate and right tibia-fibula bone were removed and soft tissues were completely dissected. Bone samples were dried for two weeks at room temperature. Dry-Ashing was performed at $600^{\circ} \mathrm{C}$ for $8 \mathrm{~h}$ and samples were oxidized for $16 \mathrm{~h}$ at $100^{\circ} \mathrm{C}$ bath with a mixture of nitric acid $65 \%$ and perchloric acid $70 \%$ with $7 / 3$ ratio, there after. Bone calcium content was determined by using an AA670 Shimadzu flame atomic absorption spectrophotometer.

\subsection{Statistical Analysis}

Data were presented as mean $\pm \mathrm{SD}$. Data analysis was carried out by using one-way ANOVA and Tukey's multiple comparison tests as the post hoc (SPSS 11.5 for windows software). Differences were considered significant at $\mathrm{p}<0.05$.

\section{RESULTS}

\subsection{Calcium and Phosphate Levels in Serum and Urine}

No significant difference observed in serum calcium or phosphate levels among different groups ( $p>0.05$ ), however; an obvious hypercalciuria associated with hyperphosphaturia was present in MP group as compared to control $(p<0.001$ for both comparisons). Niacin significantly decreased urinary calcium $(p<0.001)$ and phosphate $(\mathrm{p}=0.005)$ concentrations as compared to MP group. Calcium level was still significantly higher than control $(p<0.001)$, while phosphate decreased even to a lower level than control $(\mathrm{p}=0.005)$. Data are summarized in Table 1. 
Tahoora Shomali and Ali Fakhrzad / American Journal of Pharmacology and Toxicology 8 (2): 73-77, 2013

Table 1. Serum and urinary calcium and phosphate levels of rats in different groups at the end of 4th week of treatment

\begin{tabular}{lllr}
\hline & Control & MP & MP+N \\
\hline Serum calcium (mg/dl) & $9.26 \pm 1.3$ & $10.66 \pm 1.23$ & $9.58 \pm 0.71$ \\
Serum phosphate (mg/dl) & $5.52 \pm 0.81$ & $6.38 \pm 0.79$ & $5.43 \pm 0.57$ \\
Urinary calcium (mg/dl) & $4.04 \pm 0.54$ & $11.03 \pm 1.13^{*}$ & $7.63 \pm 0.74^{* * \#}$ \\
Urinary phosphate (mg/dl) & $62.84 \pm 15.9$ & $125 \pm 20.17^{*}$ & $29.75 \pm 5.42^{*}, \#$ \\
\hline
\end{tabular}

Control: Normal saline; MP: Methylprednisolone, $3.5 \mathrm{mg} \mathrm{kg}^{-1}$ five days a week; MP+N: Methylprednisolone $3.5 \mathrm{mg} \mathrm{kg}^{-1}$ five days a week + niacin $200 \mathrm{mg} \mathrm{kg}^{-1}$ daily. ${ }^{*}$ and ${ }^{\#}$ signs are used to demonstrate significant difference with control and MP group respectively $(\mathrm{p}<0.05)$

Table 2. Calcium content of tibia-fibula bone and forth lumbar vertebrate of rats in different groups at the end of 4th week of treatment

\begin{tabular}{llll}
\hline & Control & MP & MP+N \\
\hline Tibia-fibula bone $\left(\mathrm{mg} / \mathrm{cm}^{3}\right)$ & $297 \pm 31$ & $289 \pm 12$ & $297 \pm 15$ \\
Forth lumbar vertebrate $\left(\mathrm{mg} / \mathrm{cm}^{3}\right)$ & $298 \pm 44$ & $280 \pm 16$ & $307 \pm 17$ \\
\hline
\end{tabular}

Control: Normal saline; MP: Methylprednisolone, $3.5 \mathrm{mg} \mathrm{kg}^{-1}$ five days a week; MP+N: Methylprednisolone $3.5 \mathrm{mg} \mathrm{kg}^{-1}$ five days a week + niacin $200 \mathrm{mg} \mathrm{kg}^{-1}$ daily. No significant difference observed among groups

\subsection{Calcium Content of Forth Lumbar Vertebrate and Tibia-Fibula Bone}

No significant difference observed in calcium content of forth lumbar vertebrate or tibia-fibula bone of rats in different groups ( $\mathrm{p}>0.05)$. Data are presented in Table 2 .

\section{DISCUSSION}

Prolonged GC use induces osteoporosis; the pathogenesis of this condition is multi factorial and includes GC-induced hypercalciuria (Duzen et al., 2012). Kruse et al. (1988) observed that GCs induce hyperphosphaturia due to decreased renal phosphate reabsorption not mediated by secondary hyperparathyroidism, as well as marked hypercalciuria in children. These researchers recommended administration of hydrochlorothiazide for correcting hypercalciuria and oral phosphate for hypophosphatemia and replacement of over excreted phosphate from kidneys.

Rats were widely used as a model to study the efficacy of various treatments in the prevention of GCinduced bone loss (Li et al., 2007). In growing rats, the prevailing activity on the bone surfaces is modeling, with a linear growth which is rapid until 6 months (Erben, 1966). Our study shows that growing rats clearly exhibit hypercalciuria and hyperphosphaturia due to long term and high dose GC treatment and may be used as a model for evaluation of potential agents with effects on calcium or phosphate balance in this situation. As noted elsewhere, we did not observe significant changes in serum calcium and phosphate levels of MP treated rats. This is consistent with the findings of Wang et al. (2002) who observed that administration of MP to rats at the dosages of 2.5, 5, 10 and $20 \mathrm{mg} \mathrm{kg}^{-1}$ day $^{-1}$ for 4 weeks does not affect serum calcium and phosphorus concentrations. These reaserches did not evaluate the urinary levels of this ions. It seems that the derangement in calcium and phosphate metabolism due to MP administration is not reflected in their serum concentartion in rats.

Niacin is required at doses of $15-20 \mathrm{mg} \mathrm{day}^{-1}$ as a vitamin. However, when it is given in supraphysiological doses, exerts a variety of pharmacological effects (Lukasova et al., 2011b). Niacin administration in pharmacological doses seems to be relatively safe. Flushing and gastrointestinal symptoms such as dyspepsia, diarrhea or nausea are the most common unwanted effects of oral niacin therapy. These effects are harmless, never the less, flushing can affect patients' compliance. This effect has been reduced by using extended-release products (Kamanna et al., 2009b). Niacin, which strongly increases HDL cholesterol levels and has a well-documented clinical efficacy, has attracted new interest. Few studies are available which have addressed the effect of niacin on phosphorus metabolism. Recently, (Bostom et al., 2011) demonstrated that extended-release niacin/laropiprant (a PGD2 receptor antagonist for inhibition of flushing due to niacin) lowers serum phosphorus concentrations in diabetic patients with renal disorders. Moreover, Maccubbin et al. (2010) observed a reduction in serum phosphorus concentration of patients who have dyslipidemia and are free of advanced renal disease. As far as we know no study has addressed the effect of niacin on calcium and phosphate disturbances due to $\mathrm{GC}$ administration which establishes the rationality for our research.

We observed that niacin significantly reduces urinary calcium and phosphate levels as compared to rats treated with GC alone. The effect of niacin on urinary 
phosphate level was so strong where it was reduced to levels even lower than control without any significant change in serum phosphate level as compared to control.

Moreover, GC adiminstration did not result in a significant reduction in calcium content of forth lumbar vertebrate (as a cancellous bone) or tibia-fibula (as a cortical bone) as compared to control group, although a slight reduction was present in both bones. A possible explanation may be the relatively short period of GC treatment, where the loss of calcium from bones for compensation of serum calcium was not still detectable by flame atomic absorption. On the other hand, the effect of niacin on urinary calcium level was not associated with appreciable changes in calcium content of bones as compared to GC group, although this parameter was slightly higher in niacin treated group than GC group specially for lumbar vertebrate. Regardless of the relatively short term of the experiment, this may show that the effect of niacin on amelioration of hypercalciuria due to GC administration may be at least partly due to its plausible effects on other organs which are involved in calcium homeostasis, especially the kidneys. Although this is highly speculative and needs to be further investigated in future studies.

\section{CONCLUSION}

Niacin at its pharmacological dose can ameliorate hypercalciuria and hyperphosphaturia due to long term and high dose MP administration in growing rats without affecting bone calcium content. The possible mechanisms involved in this effect and its clinical importance needs to be clarified in further studies.

\section{ACKNOWLEDGEMENT}

Funding for this study was provided by School of Veterinary Medicine, Shiraz University, Shiraz, Iran.

\section{REFERENCES}

Bentur, L., J. Taisir and Y. Bentur, 2003. The effect of inhaled corticosteroids on the urinary calcium to creatinine ratio in childhood asthma. Therapie, 58: 313-316. DOI: $10.2515 /$ therapie:2003048

Bostom, A.G., A.A. MacLean, D. Maccubbin, D. Tipping and H. Giezek, 2011. Extended-release niacin/laropiprant lowers serum phosphorus concentrations in patients with type 2 diabetes. J. Clin. Lipidol., 5: 281-287. PMID: 21784373
Duzen, O., R. Erkoc, H. Begenik, Y.U. Soyoral and M.N. Aldemir, 2012. The course of hypercalciuria and related markers of bone metabolism parameters associated with corticosteroid treatment. Ren Fail, 34: 338-342. PMID: 22260330

Erben, R.G., 1966. Trabecular and endocortical bone surfaces in the rat: Modeling or remodeling. Anat. Rec., 246: 39-46. PMID: 8876822

Faggiano, A., R. Pivonello, D. Melis, M. Filippella and C. Di Somma, 2003. Nephrolithiasis in Cushing's disease: Prevalence, etiopathogenesis and modification after disease cure. J. Clin. Endocrinol. Metab., 88: 2076-2080. PMID: 12727957

Faggiano, J.W., N.D. Adams, J. Lemann, R.W. Gray and C.J. Thomas, 1982. Vitamin-D metabolites and parathyroid hormone in Cushing's syndromerelationship to calcium and phosphorus homeostasis. J. Clin. Endocrinol. Metab., 54: 10391044. PMID: 6977549

Gille, A., E.T. Bodor, K. Ahmed and S. Offermanns, 2008. Nicotinic acid: Pharmacological effects and mechanisms of action. Annu. Rev. Pharmacol. Toxicol., 48:79-106. PMID: 17705685

Hess, R.S., P.H. Kass and C.R. Ward, 1998. Association between hyperadrenocorticism and development of calcium-containing uroliths in dogs with urolithiasis. J. Am. Vet. Med. Assoc., 212: 1889-1891. PMID: 9638187

Kamanna, V.S., S.H. Ganji and M.L. Kashyap, 2009a. Niacin an old drug rejuvenated. Curr. Atheroscler. Rep., 11: 45-51. PMID: 19080727

Kamanna, V.S., S.H. Ganji and M.L. Kashyap, 2009 b. The mechanism and mitigation of niacin-induced flushing. Int. J. Clin. Pract., 63: 1369-1377. DOI: 10.1111/j.1742-1241.2009.02099.x

Kruse, K., M. Busse, U. Kracht, U. Kruse and K. Wohlfart, 1988. Disorders of calcium and bone metabolism in glucocorticoid treatment. Monatsschr Kinderheilkd., 136: 237-242. PMID: 2841591

Li, Y., Y. Li and W. Yang, 2007. Preventive Effects of nitroglycerine on glucocorticoid-induced osteoporosis in growing rats. J. Huazhong Univ. Sci. Technol. Med. Sci., 27: 528-531. PMID: 18060627

Lukasova, M., C. Malaval, A. Gille, J. Kero and S. Offermanns, 2011a. Nicotinic acid inhibits progression of atherosclerosis in mice through its receptor GPR109A expressed by immune cells. J. Clin. Invest., 121: 1163-1173. PMID: 21317532 
Lukasova, M., J. Hanson, S. Tunaru and S. Offermanns, 2011b. Nicotinic acid (niacin): New lipidindependent mechanisms of action and therapeutic potentials. Trends Pharmacol. Sci., 32: 700-707. PMID: 21944259

Maccubbin, D., D., Tipping, O., Kuznetsova, W.A., Hanlon and A.G., Bostom, 2010. Hypophosphatemic effect of niacin in patients without renal failure: A randomized trial. Clin. J. Am. Soc. Nephrol., 5: 582589. PMID: 20299362

Muller, D., H. Mehling, B. Otto, R. Bergmann-Lips and F. Luft et al., 2007. Niacin lowers serum phosphate and increases HDL cholesterol in dialysis patients. Clin. J. Am. Soc. Nephrol., 2: 1249-1254. PMID: 17913971

Offermanns, S., S.L. Colletti, T.W. Lovenberg, G. Semple and A. Wise, 2011. International Union of Basic and Clinical Pharmacology. LXXXII: Nomenclature and Classification of Hydroxycarboxylic Acid Receptors (GPR81, GPR109A and GPR109B). Pharmacol. Rev., 63: 269-290. PMID: 21454438

Ramsey, I.K., A. Tebb, E. Harris, H. Evans and M.E. Herrtage, 2005. Hyperparathyroidism in dogs with hyperadrenocorticism. J. Small Anim. Pract., 46: 531-536. PMID: 16300114
Smets, P., E. Meyer, B. Maddens and S. Daminet, 2010. Cushing's syndrome. Glucocorticoids and the kidney. Gen. Comput. Endocrinol., 169: 1-10. PMID: 20655918

Vrtovsnik, F., M. Jourdain, G. Cherqui, J. Lefebvre and G. Friedlander, 1994. Glucocorticoid inhibition of $\mathrm{Na}-\mathrm{Pi}$ cotransport in renal epithelial cells is mediated by protein kinase C. J. Biol. Chem., 269: 8872-8877. PMID: 8132623

Wang, Y., M. Ohtsuka-Isoya, P. Shao, S. Sakamoto and H. Shinoda, 2002. Effects of methylprednisolone on bone formation and resorption in rats. Jpn. J. Pharmacol., 90: 236-46. PMID: 12499578

Wu, B.J., L. Yan, F. Charlton, P. Witting and P.J. Barter, 2010. Evidence that niacin inhibits acute vascular inflammation and improves endothelial dysfunction independent of changes in plasma lipids. Arterioscler. Thromb. Vasc. Biol., 30: 968-975. DOI: 10.1161/ATVBAHA.109.201129 